\title{
Novel Real-Time Tremor Transduction Technique for Microsurgery
}

\author{
Damian Tomlin ${ }^{1,2}$, Jeffrey Wallace ${ }^{1,2}$, Ralph Etienne-Cummings ${ }^{1,3}$, and \\ Nitish Thakor ${ }^{1,2}$ \\ ${ }^{1}$ Engineer Research Center for Computer Integrated Surgical Systems and Technology, \\ Baltimore, MD, USA \\ 2 Johns Hopkins University, Department of Biomedical Engineering, Baltimore, MD, USA \\ nthakor@bme.jhu.edu \\ ${ }^{3}$ Johns Hopkins University, Department of Electrical and Computer Engineering, \\ Baltimore, MD, USA \\ retienne@jhu.edu
}

\begin{abstract}
Physiological tremor is one of the limiting factors to the scale on which a microsurgeon can operate. The ability to correctly determine the amount of tremor a surgeon has is an invaluable tool both for tremor cancellation and for surgical training. For this reason we have developed a novel tremor transducer using a custom built VLSI motion detection chip connected to the surgical microscope. The chip detects and measures the magnified motion of a microsurgical tool tip under the microscope. This innovative design offers several advantages over conventional methods. It is nonintrusive to the surgeon - not interfering with current microsurgical set-ups; it outputs real-time tremor data, and is small and inexpensive. The system has been implemented in an experimental set-up for analysis of the factors that aggravate tremor. Results presented, include the use of the system as a audio feedback mechanism for tremor reduction - eliciting up to a $16 \%$ reduction in tremor.
\end{abstract}

\section{Introduction}

Microsurgery involves the performance of surgery on very small blood vessels, nerves and other tissue under microscope magnification. The most dexterous of microsurgeons gain their accolades from their ability to perform very precise procedures on smaller and smaller tissue. As surgeons and clinicians seek to push the limits of microsurgical practice, they encounter several problems - tremor for one. The effect of tremor on microsurgical practice becomes quickly obvious when one looks at the relative scale of microsurgery. With tremor amplitudes on the order of $133 \mu \mathrm{m}$ [1] working on a $100-\mu \mathrm{m}$ blood vessel can be quite a formidable task.

Tremor is broadly defined as any involuntary, approximately rhythmic, and roughly sinusoidal movement [3]. More specifically physiologic tremor is defined as a normal involuntary motion accompanying all postures and movements. There are two rhythmic components of physiologic tremor: an 8 to $12-\mathrm{Hz}$ component and a mechanical-reflex component. The frequency of physiologic tremor is classically 
stated to be at $10 \mathrm{~Hz}$ but it tends to vary depending on the part of the body from which it is recorded. Although there is no conclusive evidence to support a relationship between the genesis of $8-$ to $12-\mathrm{Hz}$ tremor and visual feedback, investigations by Isokawa-Akesson and Komisaruk [8] support a correlation between the amplitude and frequency of 8- to $12-\mathrm{Hz}$ tremor and visual feedback.

The methods developed in this paper were used to investigate a correlation between 8 - to $12-\mathrm{Hz}$ tremor and audio feedback. This correlation was hypothesized by Harwell and Ferguson [17], who report that with audio feedback of tremor magnitude microsurgical trainees were able to determine which factors aggravate their tremor and make adjustments to minimize it. In effect, the system developed here provides substantial data in the exploration of ways to reduce surgical tremor.

\section{System Design and Implementation}

\subsection{Prior Tremor Sensing Techniques}

Prior tremor sensing techniques include Hall Effect sensors. The Microsurgery Advanced Design Laboratory Stability, Activation, and Maneuverability tester (MADSAM) is one such system. The MADSAM uses a magnetic field sensor, an analog signal capture board, and customized software routines to correlate magnetic field strength with physical location. The MADSAM can record the position of a small ceramic magnet affixed to a microsurgical instrument, at an accuracy of 1 micron [16]. Norman et al [15] discussed the measurement of tremor using laserbased transducers. The measurement principle of a laser transducer is based upon the Doppler effect. Monochromatic laser light, scattered back from a vibrating target, undergoes a frequency shift proportional to the velocity of the target. The frequency of the back-scattered beam is frequency modulated at the so-called Doppler frequency, which is directly proportional to the velocity of the target. Accelerators have also been used in tremor-sensing systems. They are highly favored because acceleration is widely considered as a more relevant variable for tremor than is velocity or displacement [15]. Gomez-Blanco et al [14] developed a method for sensing hand tremor in a vitreoretinal microsurgical instrument by attaching three accelerometers to the instrument. Hall Effect sensors and laser-based transducers are not practical for intraoperative use because of their size and mode of operation, and accelerometers intrude on the surgeon by adding some weight to his tool. Our system fills the void created by these different tremor-sensing modalities by offering a completely non-intrusive and easily implemented design.

\subsection{Design Criteria}

The system is designed to be non-intrusive, to offer the requisite frequency response and artifact rejection, and to be cost-effective. The technique employed does not interfere with the current setup for performing microsurgery. It causes minimal obtrusion to the surgical field. This allows for a test-bed setup that is not cumbersome and that does not introduce undesirable interference to the experiment. The system, as described below, involves only a simply connection to the video output port of the surgical microscope. 
Sampling frequency restrictions are also be satisfied. The characteristic frequency of physiologic tremor, on the order of $8-12 \mathrm{~Hz}$, dictates that the sampling frequency of any transducer system must be, at the bare minimum, $24 \mathrm{~Hz}$ to comply with the Nyquist sampling theorem and avoid aliasing problems. For our experiment we used a sampling frequency of $250 \mathrm{~Hz}$, which is well above the Nyquist limit.

\subsection{Stereomicroscope, DAQ System}

Figure 1 shows a schematic of the full system. The shiny tool tip reflects light from the microscope light source. Against a non-reflective background the tool tip is easily identified and differentiated from any other movement within the field of view. This ensures that the chip detects only the tool-tip's motion. Furthermore, during experiments, the tool tip is the only moving object in focus underneath the microscope. The motion of the tool tip is magnified through the microscope and translated onto the surface of the motion detection chip.

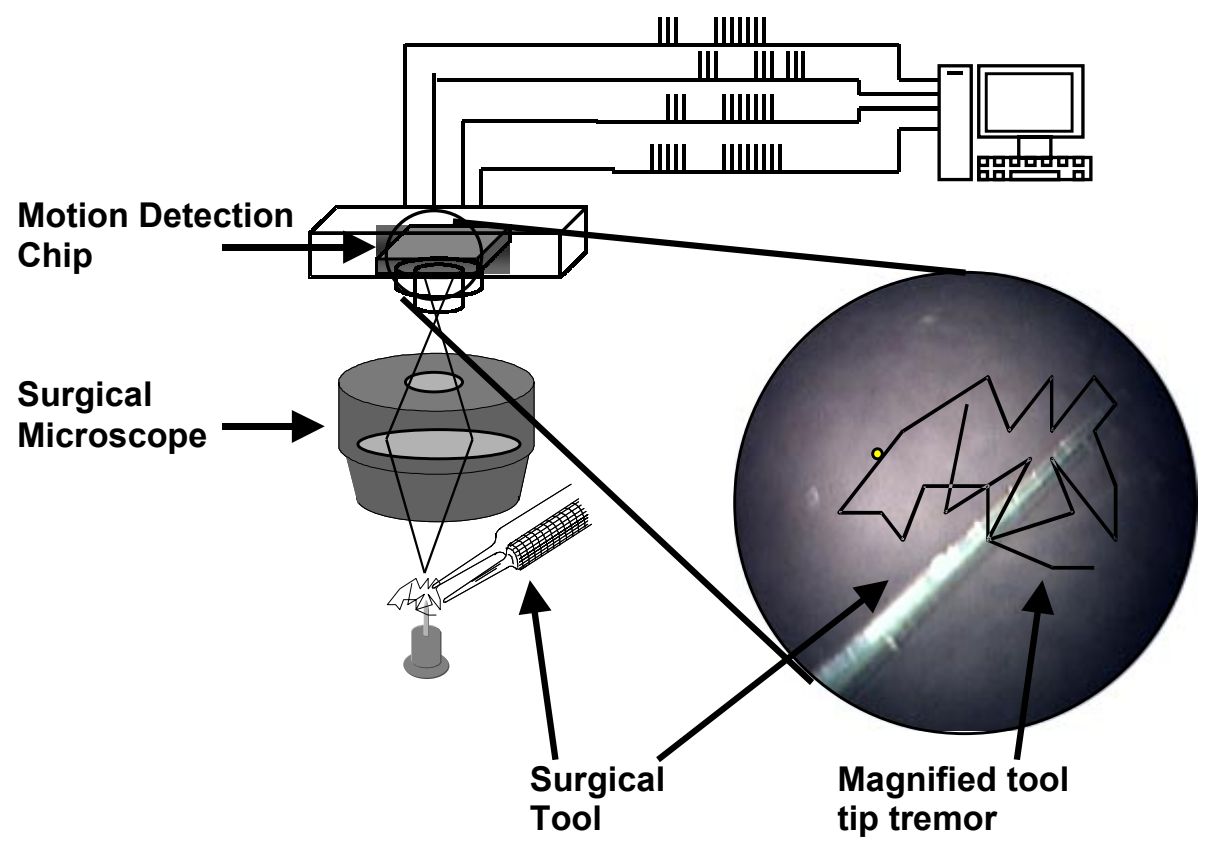

Figure 1: Schematic of the tremor analysis system. The magnified motion of the surgical tool is detected by the motion detection chip. The speed and direction of motion is reported to the data acauisition svstem. A view of the surgical tool from the chin's nersnective is also shown.

The chip has four output lines from the fovea (See Section 2.3). These lines correspond to the Left, Right, Up and Down directions in the plane of the chip. Each line outputs a $5 \mathrm{~V}$ pulse-train whenever the chip detects an object moving in its direction. In LabVIEW (a visual programming language from National Instruments, Austin, TX), the number of pulses from each output line is averaged over a specific time period. This average (or the density of the pulse-train) is proportional to the 
speed of the object the chip detects. The average number of Left pulses is subtracted from the average number of Right pulses to yield the X-component of tremor velocity. Similarly, the Y-component is obtained by subtracting the average number of Down pulses from Up pulses. This way a vector representing the velocity of the tremor signal is obtained. The acceleration signal is obtained by a five-point derivative of each component of velocity and, in turn, a vector addition of both resulting acceleration components. The equation below shows the expression used to compute the derivative.

$$
d f=C_{1} f(n)+C_{2} f(n-1)-C_{2} f(n-2)-C_{1} f(n-3)
$$

where $\mathrm{C}_{1}=1.08144$ and $\mathrm{C}_{2}=2.69869$ are coefficients determined from a Gaussian derivative detailed in [19].

The motion detection chip, housed in a $3 \times 2 \times 1$ inch box is connected to the video port
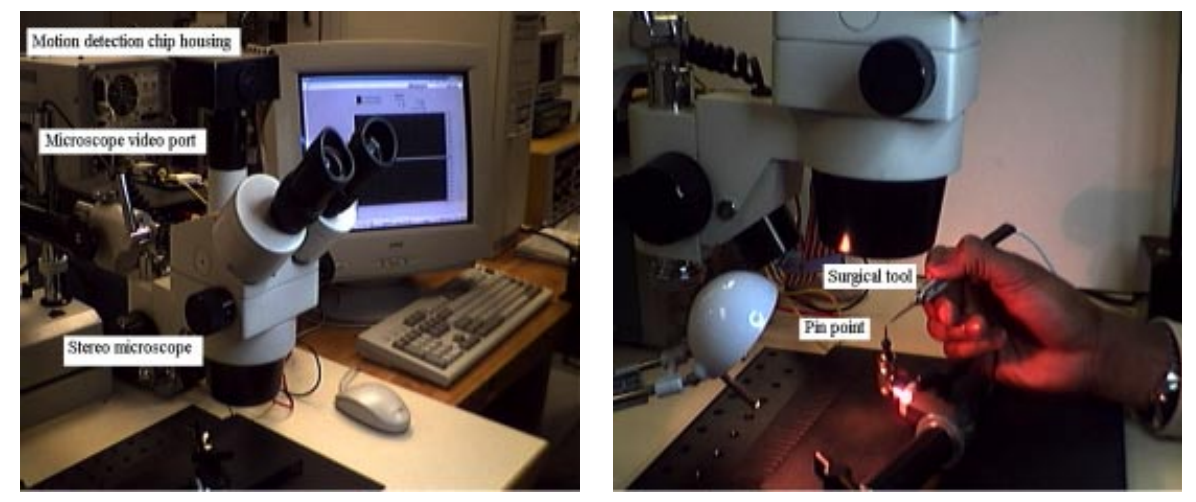

Figure 2. Picture of the setup. The magnified motion of the surgical tool is detected by the motion detection chip connected at the video port of the microscope.

of the microscope through a two-inch C-mount connector (Figure 2). The "focus" knob of the microscope is used to account for the two-inch focal length discrepancy caused by the introduction of the connector. The camera is connected to a PCI-MIO16E-4 data acquisition card (National Instruments, Austin TX), which is, in turn, accessed from LabVIEW 5.1 software.

\subsection{VLSI Motion Detection Chip}

Figure 3 shows a picture of the motion detection chip used in the system. The chip is organized into a foveal region and a peripheral region, similar to primate biological systems. It uses compact focal-plane processing to allow for two-dimensional tracking [12]. When a target enters the fovea, an edge detection algorithm determines the edge of the target. The direction of motion of the target is determined by which neighboring pixel the edge reappears after disappearing from one pixel. The motion detection circuit in the fovea produces the pulse train on the Left, Right, Up and Down global motion lines when a target moves away from the center in any of these directions. The chip has two processing stages: phototransduction and edge detection. 


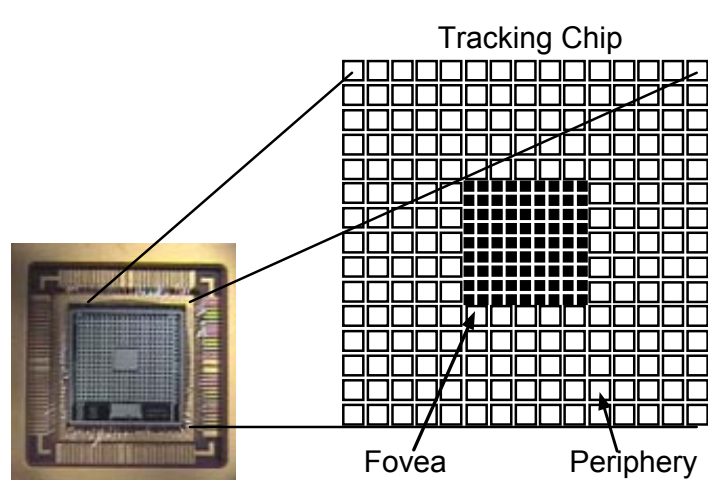

Figure 3: Motion detection chip. The fovea is towards the center of the chip, and the periphery immediately surrounds the fovea.
The photo-transduction stage contains the phototransistors and range compression circuits, and the edge detection stage computes the edge of the target. A number of issues were considered to ensure that the chip could satisfy the requirements for a tremor transducer. The photoreceptors (phototransistors) and the edge detection circuitry must be able to correctly determine the edge of the target, and the fovea must be able to report the correct information about

the motion of the target. The chip must also possess adequate speed sensitivity. Each of these criterions was tested independently. See [12] for chip performance test results. The chip is operational for ambient intensities ranging over 6 orders of magnitude, targets contrast as low as $10 \%$, and foveal speed ranging from 1.5 to $10 \mathrm{~K}$ pixels/s.

\section{Results and Discussion}

Results are presented for the system in operation for two separate experiments: tremor acceleration magnitude versus speed of movement, and tremor acceleration magnitude with and without audio feedback. Figure 4 shows the conversion from

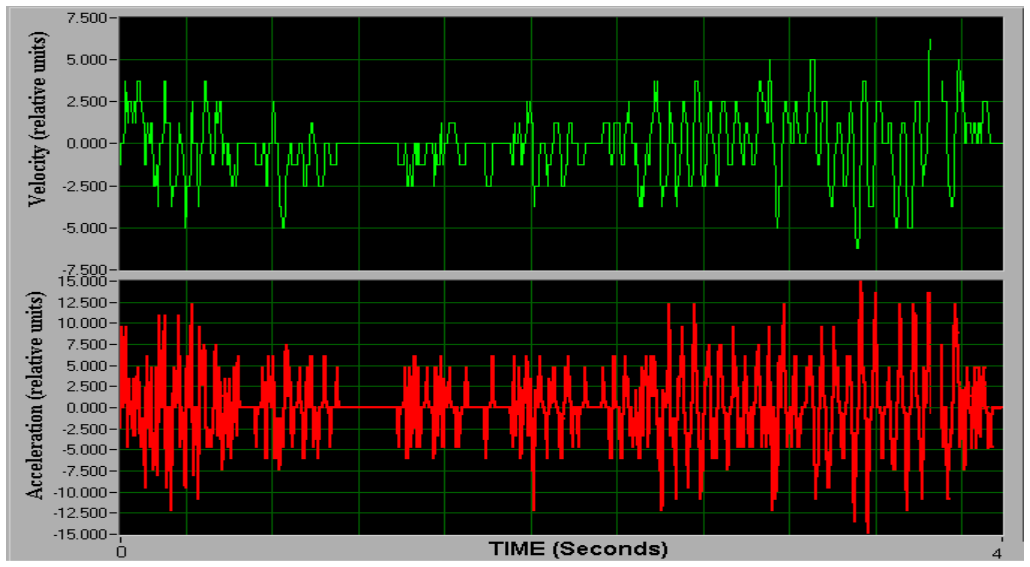

Figure 4. Top graph shows velocity in the X-direction (average Right pulses subtract minus average Left pulses) and bottom graph shows the acceleration in the $\mathrm{X}$-direction (derivative of velocity). 
velocity, as determined from the transducer, to acceleration (determined by a Gaussian derivative of the velocity) The top graph in shows the velocity of the tool tip in the X-direction and the bottom graph shows the acceleration in the X-direction. The top graph of Figure 5 shows the magnitude of the acceleration vector and the bottom graph shows a moving average over 50 samples. The magnitude of the acceleration vector is proportional to the amount of tremor present.

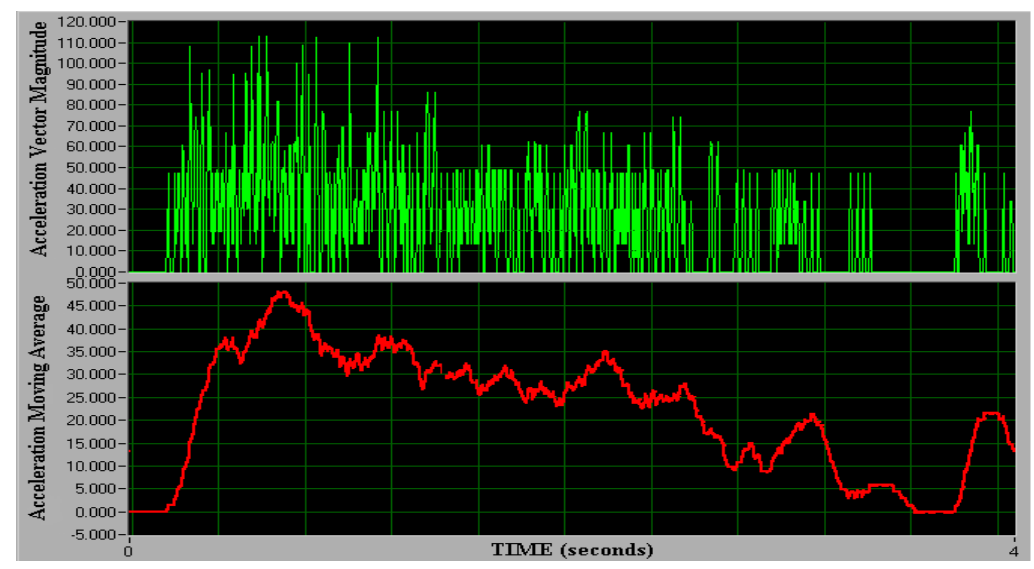

Figure 5. Top graph shows the magnitude of the acceleration vector (vector addition of $\mathrm{X}$ and $\mathrm{Y}$ components). Bottom graph shows a moving average over 50 samples.

Figure 6 shows results obtained from the system when measuring tremor against a variation in the speed of movement. In this experiment the subject is asked to move a microsurgical tool back and forth in the microscope's field of view at varying frequencies of motion. The speed of oscillation is kept by a metronome. The mean tremor acceleration is recorded for frequencies ranging from 1 to $5 \mathrm{~Hz}$. Figure 6a

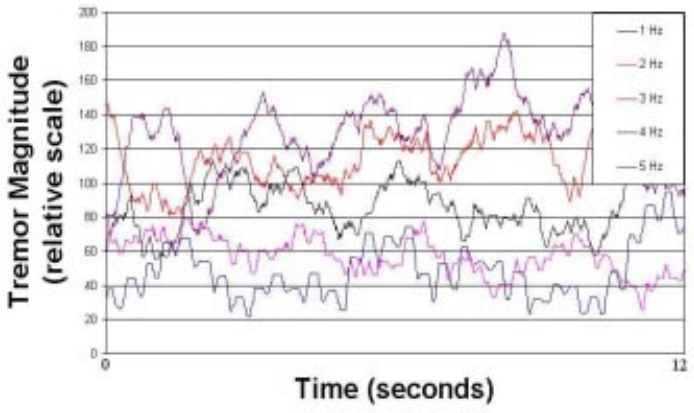

(a)

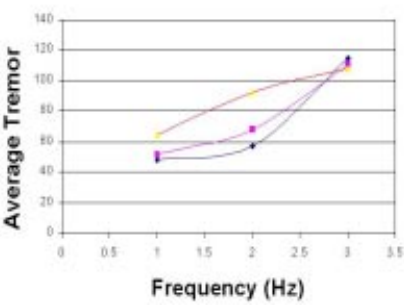

(b)

Figure 6. Tremor recorded for voluntary oscillation along a line. The subject is asked to move a microsurgical tool back and forth in the microscope field of view at varying frequency of motion. The frequency (and thus the speed) is kept by a metronome. The mean acceleration recorded increases nearly linearly with increase in the frequency of oscillation. 
shows that at each consecutive increase in frequency, the mean tremor acceleration increases. There is a nearly linear increase in tremor acceleration with frequency (Figure 6b).

Figure 7 shows the results of giving audio feedback of the magnitude of tremor to a subject with no prior surgical training. The results were generated using the Microsoft Excel Analysis of Variance (ANOVA) statistical analysis tool. The subject was asked to point the surgical tool at a pinhead under the microscope - holding his hand as steady as possible - first without audio feedback of tremor magnitude, then with audio feedback. This was repeated five times. Without audio feedback the average tremor acceleration recorded was 35.6 and with feedback it was 30.6. This is a statistically significant decrease in average tremor of $16 \%(\mathrm{P}<.05)$.

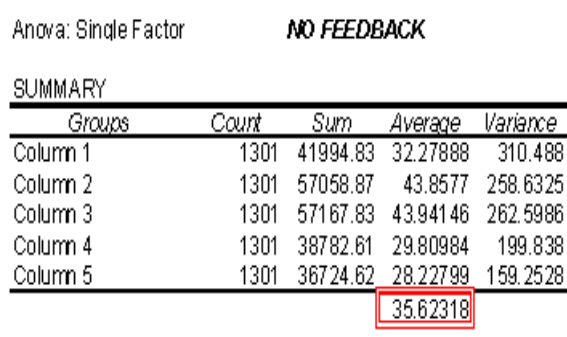

(a)

\begin{tabular}{|c|c|c|c|c|}
\hline Anowa: Single Factor & & & WITHFEEL & DBACK \\
\hline SUMMARY & & & & \\
\hline Groups & Count & Sum & Average & Variance \\
\hline Column 1 & 1301 & 45577.67 & 35.0328 & 483.5768 \\
\hline Colum 2 & 1301 & 45231.8 & 34.76695 & 334.6004 \\
\hline Colum 3 & 1301 & 15535.66 & 11.94132 & 50.27678 \\
\hline Columm 4 & 1301 & 52235.77 & 40.15048 & 124.3033 \\
\hline Column 5 & 1301 & 40723.61 & 31.30178 & 243.8863 \\
\hline & & & \begin{tabular}{|l|}
30.63867 \\
\end{tabular} & \\
\hline
\end{tabular}

(b)

Figure7. Results for audio feedback analysis. The "boxed" figures show the average tremor magnitude for 5 experiments. With feedback there is a $16.5 \%$ decrease in tremor magnitude. (a) the average tremor acceleration is recorded over a 30 -second time period for a subject holding the surgical tool steady at one single point. (b) The experiment is repeated while giving audio feedback of acceleration.

\section{Conclusions}

The key strengths of the system described here are its unique characteristic as an easily implemented alternative to conventional tremor transducers, and the minimal processing needed in its operation. It is also completely non-intrusive to the surgeon and does not interfere with the microsurgical field at all. It is therefore, an effective tremor transducer. Through our preliminary results on non-surgical personnel, we have seen that the system, through feedback pertaining to the amount of tremor, allows the individual to ergonomically adjust their posture and reduce their tremor. More experiments need to be run to determine the outcome of long-term learning with audio feedback on tremor reduction. The possible benefits of the system, however, can be seen from this initial work. Through feedback, the amount of tremor a person has can be minimized for finer, more precise movements during a procedure. This has the ability to improve upon surgical outcomes by decreasing tremor related errors and by decreasing surgery times. 


\section{References}

1. Riviere CN, Rader RS, Khosla PK. Characteristics of Hand Motion of Eye Surgeons. Prodeedings - 19th International Conference - IEEE/EMBS Oct. 30 - Nov. 2, 1997 Chicago, IL. USA.

2. Silber, SJ. Microsurgery, 1979. The Williams \& Wilkins Company, Baltimore, MD

3. Elble R, Koller W. Tremor. Baltimore, MD: Johns Hopkins University Press, 1990

4. Stiles RN. Mechanical and Neural Feedback Factors in Postural Hand Tremor of Normal Subjects. Journal of Neurophysiology 1980; 44:40-59.

5. Wade P, Gresty MA, Findley LJ. A Normative Study of Postural Tremor of the Hand. Archives of Neurology 1982; 39:358-362.

6. Elble RJ. Physiologic and Essential Tremor. Neurology 1986; 36:225-231.

7. Elble RJ, Moody C, Higgins C. Primary Writing Tremor: a form of focal dystonia? Movement Disorders 1990; 5:118-126.

8. Isokawa-Akesson M, Komisaruk BR. Tuning the Power Spectrum of Physiological Finger Tremor Frequency with Flickering light. Journal of Neuroscience Res 1985; 14:373-380.

9. Brumlik J, Yap C-B. Normal Tremor: A Comparative Study. 1970. Springfield, Ill: Charles C Thomas, pp 3-15.

10.Freund H-J. Motor Unit and Muscle Activity in Voluntary Motor Control. Physiological Reviews 1983; 63:387-436.

11.Allum JHJ, Hulliger M. Presumed reflex responses of human first dorsal interosseus muscle to naturally occurring twitch contractions of physiological tremor. Neuroscience Letters 1982; 28:309-314.

12.Etienne-Cummings R, Van der Spiegel J, Mueller P. A Foveated Silicon Retina for TwoDimensional Tracking. IEEE Trans. Circuits and System II, Vol. 47, No. 6, June 2000.

13.Reichardt W. Autocorrelation: A Principle for the Evaluation of Sensory Information by the Central Nervous System. Sensory Communication, Wiley, New York NY, 1961.

14.Gomez-Blanco M, Riviere CN, Khosla PK. Sensing Hand Tremor in a Vitreoretinal Microsurgical Instrument. CMU-RI-TR-99-39. The Robotics Institute, Carnegie Mellon University, Pittsburgh PA.

15.Norman KE, Edwards R, Beuter A. The measurement of tremor using a velocity transducer: comparison to simultaneous recordings using transducers of displacement, acceleration and muscle activity. Journal of Neuroscience Methods 1999; 92:41-54.

16.Humayun MU, Rader RS, Piermici DJ, Awh CC, de Juan E. Quantitative Measurement of the Effects of Caffeine and Propranolol on Surgeon hand Tremor. Archives of Ophthalmology 1997; 115:371-374.

17.Harwell RC, Ferguson RL. Physiologic Tremor and Microsurgery. Microsurgery 1983; 4:187-192.

18.Taylor R, Jensen P, Whitcomb L, Barnes A, Kumar R et al. A Steady-Hand Robotic System for Microsurgical Augmentation. The International Journal of Robotics Research 1999; Dec.

19.Simoncelli EP. Distributed Representation and Analysis of Visual Motion. PhD Thesis, MIT Dept. of Electrical Engineering and Computer Science, 1993; p95-97. 